\title{
Chromatin remodeling during in vivo neural stem cells differentiating to neurons in early Drosophila embryos
}

\author{
Youqiong Ye ${ }^{1,2}$, Min $\mathrm{Li}^{1,2}$, Liang $\mathrm{Gu}^{1}$, Xiaolong Chen ${ }^{1}$, Jiejun Shi ${ }^{1}$, Xiaobai Zhang ${ }^{\star, 1}$ and Cizhong Jiang ${ }^{\star, 1}$
}

Neurons are a key component of the nervous system and differentiate from multipotent neural stem cells (NSCs). Chromatin remodeling has a critical role in the differentiation process. However, its in vivo epigenetic regulatory role remains unknown. We show here that nucleosome depletion regions (NDRs) form in both proximal promoters and distal enhancers during NSCs differentiating into neurons in the early Drosophila embryonic development. NDR formation in the regulatory regions involves nucleosome shift and eviction. Nucleosome occupancy in promoter NDRs is inversely proportional to the gene activity. Genes with promoter NDR formation during differentiation are enriched for functions related to neuron development and maturation. Active histone-modification signals ( $\mathrm{H} 3 \mathrm{~K} 4 \mathrm{me} 3$ and $\mathrm{H} 3 \mathrm{~K} 9 \mathrm{ac}$ ) in promoters are gained in neurons in two modes: de novo establishment to high levels or increase from the existing levels in NSCs. The gene sets corresponding to the two modes have different neuronrelated functions. Dynamic changes of $\mathrm{H} 3 \mathrm{~K} 27 \mathrm{ac}$ and $\mathrm{H} 3 \mathrm{~K} 9 \mathrm{ac}$ signals in enhancers and promoters synergistically repress genes associated with neural stem or progenitor cell-related pluripotency and upregulate genes associated with neuron projection morphogenesis, neuron differentiation, and so on. Our results offer new insights into chromatin remodeling during in vivo neuron development and lay a foundation for its epigenetic regulatory mechanism study of other lineage specification.

Cell Death and Differentiation (2017) 24, 409-420; doi:10.1038/cdd.2016.135; published online 18 November 2016

Neurons are a major and important cell type of the nervous system. Neurons differentiate from multipotent precursors called neural stem cells (NSCs). This indicates that an extensive epigenomic change occurs during differentiation. ${ }^{1}$ Epigenomic signatures, including chromatin accessibility, histone modifications (HMs), and DNA methylation, regulate the gene activity. The abnormal epigenomic changes disrupt genes that function in neural development and consequently lead to neurological and psychiatric diseases. Therefore, it is a prerequisite to characterize the in vivo chromatin states of neurons and NSCs for the analysis of epigenomic changes during NSCs differentiating into neurons.

There are many studies on the role of chromatin remodeling in cell fate specification using in vitro cell differentiation platforms. Overall, differentiated cells have a more condensed chromatin structure than stem cells. ${ }^{2}$ A bunch of studies showed changes in histone-modification patterns in embryonic stem cell (ESC) differentiation. ${ }^{3-5} \mathrm{~A}$ recent study profiled chromatin state dynamics across 16 stages of hematopoietic differentiation. ${ }^{6}$ Global chromatin states of mouse ESCs, neural progenitor cells, and embryonic fibroblasts were reported, as well as new bivalent chromatin marks. ${ }^{7}$ Both Foxa2 and $\mathrm{H} 2 \mathrm{~A}$.Z mediate nucleosome depletion during ESCs differentiating into the endoderm. ${ }^{8}$ A recent study identified 85 core HM sites in mouse ESCs and 78 sites in neural progenitor cells. ${ }^{9}$ To date, there is no study reported focusing on chromatin dynamics during in vivo neuron development.

It is critical to use specific in vivo cell types to investigate epigenetic reprogramming during differentiation because cell cultures or in vitro differentiated cells lack the proper in vivo context. However, it is a technical challenge to isolate specific neurons and NSCs for epigenomic studies that require a large number of cells. Manual sorting ${ }^{10}$ is laborious and only useful for collecting a small number of cells. Laser capture microdissection $^{11}$ is also not suitable for isolating large number of cells required by chromatin assays. Although fluorescence-activated cell sorting (FACS) can isolate large number of cells, it requires molecular markers specific to the cell type of interest that are not always available. FACS requires harsh treatment of the tissue and does not work efficiently in complex tissues such as the brain. As such, FACS is not optimal for genome-wide epigenomic profiling. Owing to an alternate approach, INTACT (isolation of nuclei tagged in specific cell types), ${ }^{12}$ it introduces into Drosophila genome a nuclear targeting fusion (NTF) gene consisting of 3xFLAG, BLRP (biotin ligase recognition peptide, a preferred substrate for BirA), mCherry, and RanGap (expressed in the cytoplasm and outer nuclear envelope). The NTF gene is expressed under the control of a certain cell-specific gene promoter. Therefore, INTACT can capture cell-type-specific nuclei

\footnotetext{
${ }^{1}$ Department of Clinical Laboratory Medicine, Shanghai Tenth People's Hospital of Tongji University, Shanghai Key Laboratory of Signaling and Disease Research, The School of Life Sciences and Technology, The Collaborative Innovation Center for Brain Science, Tongji University, Shanghai 200092, China

*Corresponding author: C Jiang or X Zhang, Department of Clinical Laboratory Medicine, Shanghai Tenth People's Hospital of Tongji University, Shanghai Key Laboratory of Signaling and Disease Research, The School of Life Sciences and Technology, The Collaborative Innovation Center for Brain Science, Tongji University, 1239 Siping Road, Shanghai 200092, China. Tel: +86 216598 1193; Fax: +86 216598 1041; E-mail: czjiang@tongji.edu.cn or zhangxb@tongji.edu.cn

${ }^{2}$ These authors contributed equally to this work.

Abbreviations: NSC, neural stem cell; NDR, nucleosome depletion region; HM, histone modification; ESC, embryonic stem cell; FACS, fluorescence-activated cell sorting; INTACT, isolation of nuclei tagged in specific cell types; NTF, nuclear targeting fusion; AEL, after egg laying; TSS, transcription start site; DEG, differentially expressed gene; GSEA, gene set enrichment analysis; GO, gene ontology

Received 16.7.16; revised 06.10.16; accepted 18.10.16; Edited by RA Knight; published online 18.11.2016
} 
suitable for gene expression, epigenomic, and proteomic profiling within a tissue through affinity purification of the NTF protein. ${ }^{13-15}$ It allows the study of distinct cell types at different stages of development.

Here we applied INTACT in Drosophila to isolate NSCs and neurons for their epigenome profiling including transcriptome, genome-wide nucleosome occupancy, and global core HM signals. Epigenomic analyses show that nucleosome depletion regions (NDRs) form in both promoters and enhancers through nucleosome shift and eviction. Nucleosome occupancy in promoter NDRs is inversely proportional to the gene activity. Genes with promoter NDR formation during differentiation are enriched for neuron development and maturation. Active H3K4me3 and H3K9ac are deposited in the promoters of genes in neurons related to neuronal functions in two modes: de novo establishment or increase from existing levels in NSCs. In addition, changes of H3K27ac and H3K9ac in promoters and enhancers synergistically upregulate genes with functional enrichment for neuron differentiation and downregulate genes with functional enrichment for neural progenitor cell-related pluripotency.

\section{Results}

Affinity purification of NSC and neuronal nuclei. To apply the INTACT ${ }^{15}$ method to isolate neuronal nuclei, we generated a Drosophila strain containing Elav-Gal4> UASNTF (Elav>NTF). Elav is the commonly used neuronal marker gene. ${ }^{16,17}$ The neuronal nuclei were then affinity purified from stage 15 to 16 embryos (12-14 h AEL) using anti-Flag-coated magnetic beads (Figures $1 \mathrm{a}$ and $\mathrm{b}$ ). Similarly, we generated a Drosophila strain containing Sca-Gal4 $>$ UAS-NTF (Sca $>$ NTF). The promoter of NSC marker gene, $S c a,{ }^{18}$ drives the specific expression of NTF gene in the nuclear envelope of NSCs. Therefore, NSC nuclei were collected from stage 11 embryos (5-7 h AEL) through affinity purification. Moreover, the affinity-purified neuronal and NSC nuclei reached a purity of $98 \pm 2 \%$ and $90 \pm 3 \%$, respectively (scored $>100$ nuclei, average of three experiments each) (Supplementary Figure S1A).

We further examined expression profiles of different tissuespecific genes that were determined by RNA in situ hybridization by Berkeley Drosophila Genome Project. ${ }^{19}$ The results show that expression levels of neuronal genes are significantly higher compared with other tissue-specific genes in neuronal nuclei (Figure 1c). Similarly, expression levels of NSC-specific genes are significantly higher than other tissue-specific genes in NSC nuclei (Supplementary Figure S1B). Consistently, the active $\mathrm{HM}$ signals (H3K4me3, H3K27ac and H3K9ac) in promoter regions ( $\pm 1 \mathrm{~kb}$ of transcription start sites (TSSs)) of neuronal genes are significantly higher than other tissuespecific genes in the neuronal nuclei, whereas the repressive HM signals (H3K27me3) are opposite (Figure 1d). The distribution pattern of $\mathrm{HM}$ signals in promoters is the same as that in NSC nuclei (Supplementary Figure S1C). These results further confirmed the purity of the isolated nuclei and appropriateness of purified nuclei for expression and chromatin profiling.
Expression profiles of isolated NSC and neuronal nuclei. We exploited RNA-seq to analyze gene expression changes during NSCs differentiating into neurons. Gene set enrichment analysis shows that genes associated with the stemness of neural progenitor cells maintain high expression levels in NSCs and are significantly downregulated in neuronal cells, whereas genes associated with neuronrelated functions are significantly upregulated during differentiation (Supplementary Figure S2A). There are a total of 2131 significantly differentially expressed genes (DEGs) in neuronal cells, of which 432 are downregulated genes and 1699 are upregulated genes (Figure 2a). The NSC marker gene, Sca, is significantly downregulated, whereas the neuronal marker gene, Elav, is upregulated during the development of NSCs to neurons (Figure 2b). Gene ontology (GO) functional annotation of the DEGs found that the downregulated DEGs are mainly enriched for neural progenitor cell-related functions: cell cycle, cell division, DNA repair, cell fate commitment, neuroblast fate determination, and so on (Supplementary Figure S2B). Conversely, the upregulated DEGs have enrichment for neuron-related functions such as neuron development, neuron projection morphogenesis, axonogenesis, and so on (Figure 2c). These results indicate an extensive change in gene expression program to satisfy the different functions of these two neural cell types during differentiation.

Global nucleosome positioning dynamics during differentiation. We next explored nucleosome positioning dynamics during differentiation. We scanned the genome with a 500-bp scan window (approximately three nucleosomes plus two linker regions) and calculated read count in each window as the nucleosome occupancy. Nucleosome occupancy changes spread out across the genome during differentiation and are statistically significant (Supplementary Figure S3A). We obtained consistent results using 150-bp (mononucleosome) and 300-bp (approximately dinucleosome plus a linker region) scan window (data not shown).

To examine nucleosome positioning dynamics, we predicted genome-wide nucleosome positions using GeneTrack, ${ }^{20}$ and obtained 523516 nucleosomes in NSCs and 514910 nucleosomes in neurons. The majority of nucleosomes (>92\%) shift by 1 to 126 bp and only $\sim 2 \%$ of nucleosomes do not shift during differentiation. In total, 5.3\% of nucleosomes are lost in neurons, whereas $4.5 \%$ of nucleosomes are newly formed in neurons (Figure 3a). Interestingly, nucleosome gain and loss are enriched in promoters (Figure 3b). This implies that nucleosome remodeling in promoters may have a critical role in the transition of gene expression program during differentiation. The genes with nucleosome gain or loss in promoters are provided in Supplementary Table S2.

Nucleosome depletion in promoter regions upon differentiation. To discover the role of nucleosome remodeling in promoter regions in transcription regulation during the differentiation, we focused on the changes in the nucleosome organization around TSS. There is a $-1, \mathrm{NDR},+1,+2,+3$, and so on canonical nucleosome arrangement around TSS (Figure 3c). We further clustered the nucleosome 
a
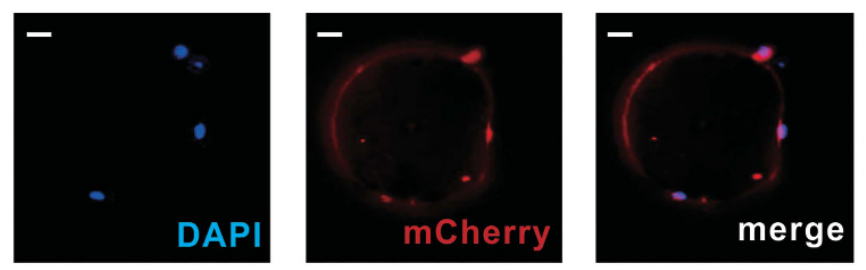

b
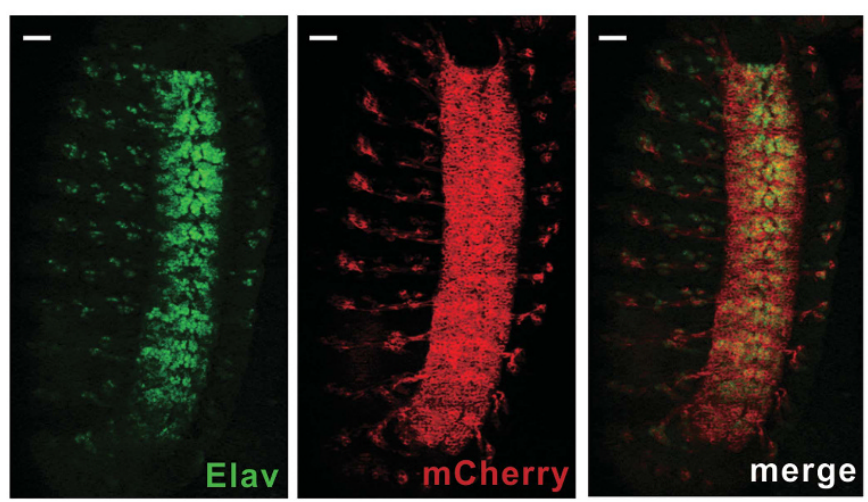

C

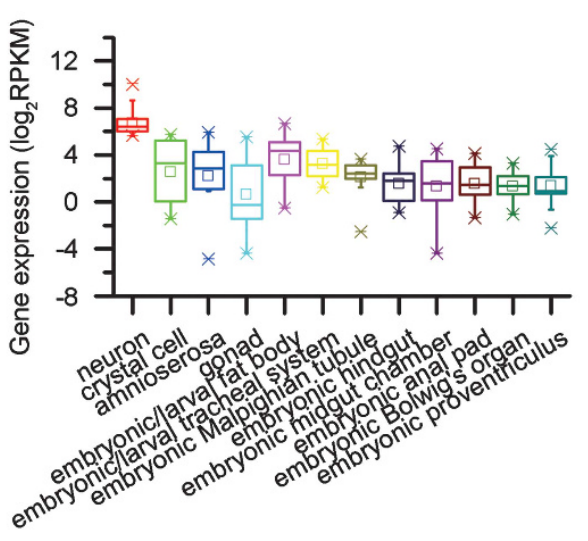

d

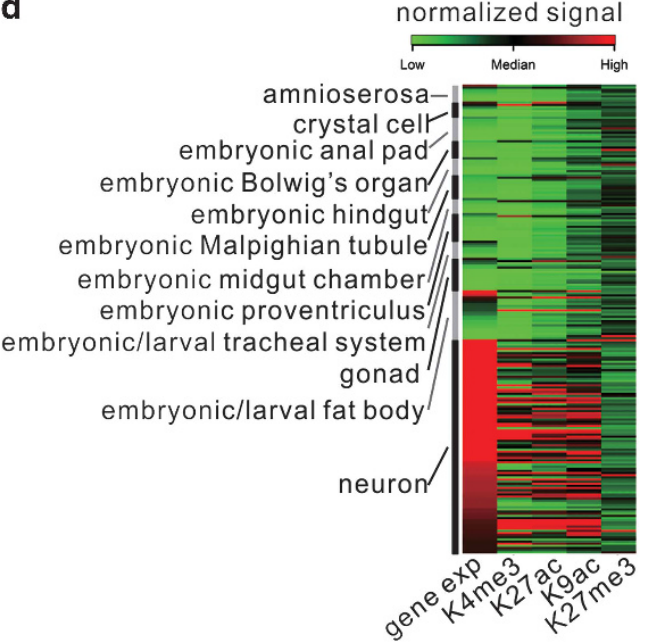

Figure 1 Affinity purification of neuronal nuclei from Drosophila melanogaster with the INTACT system. INTACT introduces into Drosophila genome an NTF gene consisting of 3xFLAG, BLRP (biotin ligase recognition peptide, a preferred substrate for BirA), mCherry, and RanGap (expressed in the cytoplasm and outer nuclear envelope). The NTF gene was expressed under the control of neuron-specific gene (Elav) promoter. (a) Three purified nuclei bound to an anti-Flag-coated bead (the large circle). Scale bar: $10 \mu \mathrm{m}$. (b) Detection of mCherry (red) epitope and endogenous Elav (green) expression in a fixed stage 15 embryo (Elav-Gal4> UAS-NTF). Scale bar: $30 \mu \mathrm{m}$. (c) The expression levels of neuron-specific genes are significantly higher compared with other tissue-specific genes in the purified neuronal nuclei. All $P$-values are $<0.01$ (Wilcoxon's rank-sum test). (d) Heatmap shows the profiles of core HMs in the promoter regions ( $\pm 1 \mathrm{~kb}$ of TSS) of different tissue-specific genes in the purified neuronal nuclei. Genes are sorted descendingly by the expression level within each tissue

organization difference around TSS. The results revealed five distinct patterns for nucleosome organization change upon differentiation (Supplementary Figure S3B). Approximately $39.3 \%$ of genes maintain a similar canonical nucleosome arrangement around TSS (Cluster 2). In contrast, the periodicity of nucleosome positioning around TSS is disrupted in a set of genes $(\sim 14.8 \%)$ in neurons by a weak peak at +4 nucleosome location. Moreover, the downstream nucleosomes starting at +5 nucleosome shift toward $5^{\prime}$ (Cluster 5). Similarly, the downstream nucleosomes starting at +3 nucleosome shift toward $5^{\prime}$ but with unbroken periodicity in another set of genes $(\sim 15.8 \%)$ in neurons (Cluster 4). The rest of the genes have periodical nucleosome positioning around TSS in both cell types. However, TSS is fully (Cluster $3, \sim 17.0 \%$ of genes) or partially (Cluster 1 , $\sim 13.1 \%$ of genes) occupied by a nucleosome in NSCs, whereas the canonical nucleosome arrangement forms around TSS in neurons leading to exposed TSS.
Interestingly, careful examination uncovered that the nucleosome occupancy in NDRs ( -200 and +50 bp to TSS) is higher in NSCs than in neurons (Figure 3c). It has been reported that nucleosome remodeling near TSS has a critical role in gene expression regulation during mouse ESCs differentiating into the lineage-committed endoderm/hepatic progenitor cells. ${ }^{8}$ Therefore, we analyzed the impact of nucleosome remodeling in NDRs on gene expression. The results show that nucleosome occupancy levels are negatively correlated with gene expression levels (Supplementary Figure S3C). Interestingly, nucleosome occupancy in NDRs in NSCs is significantly lower in NSC-specific genes than in other tissue-specific genes. Similarly, nucleosome occupancy in NDRs in neurons is significantly lower in neuron-specific genes than in other tissue-specific genes (Figure $3 d$ ). Consistently, gene expression levels are significantly higher in NSC-specific genes in NSCs and neuron-specific genes in neurons than in other tissue-specific genes (Figure 1c and Supplementary 


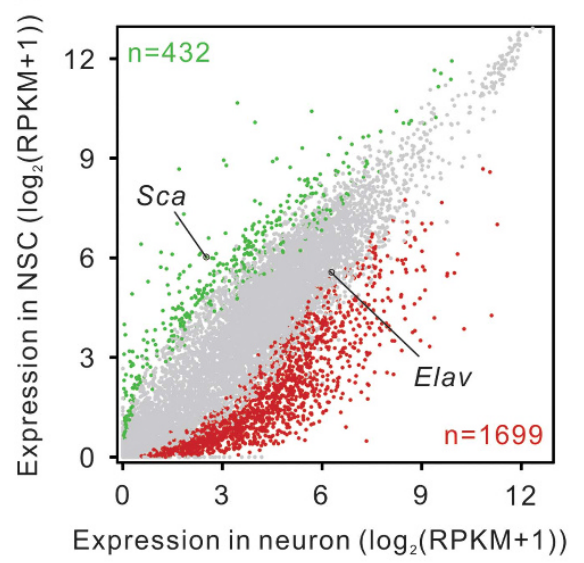

\section{b}
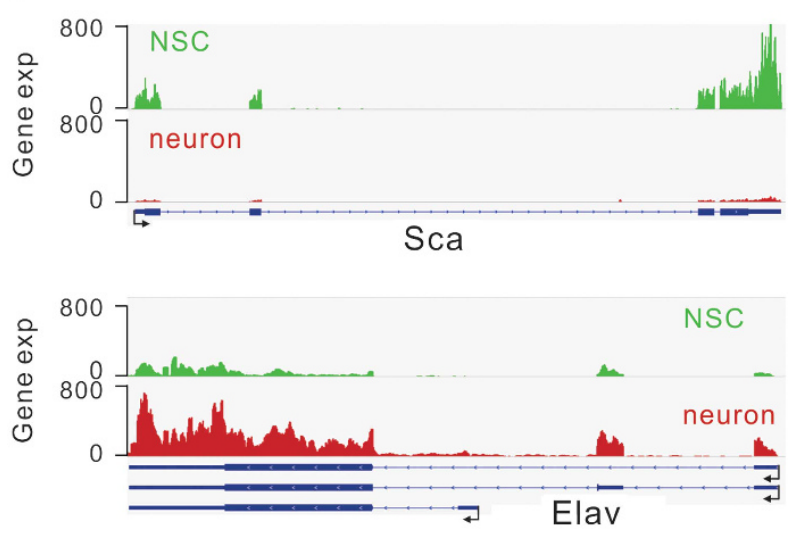

C

Enriched GO BP terms for DE genes up-regulated in neurons

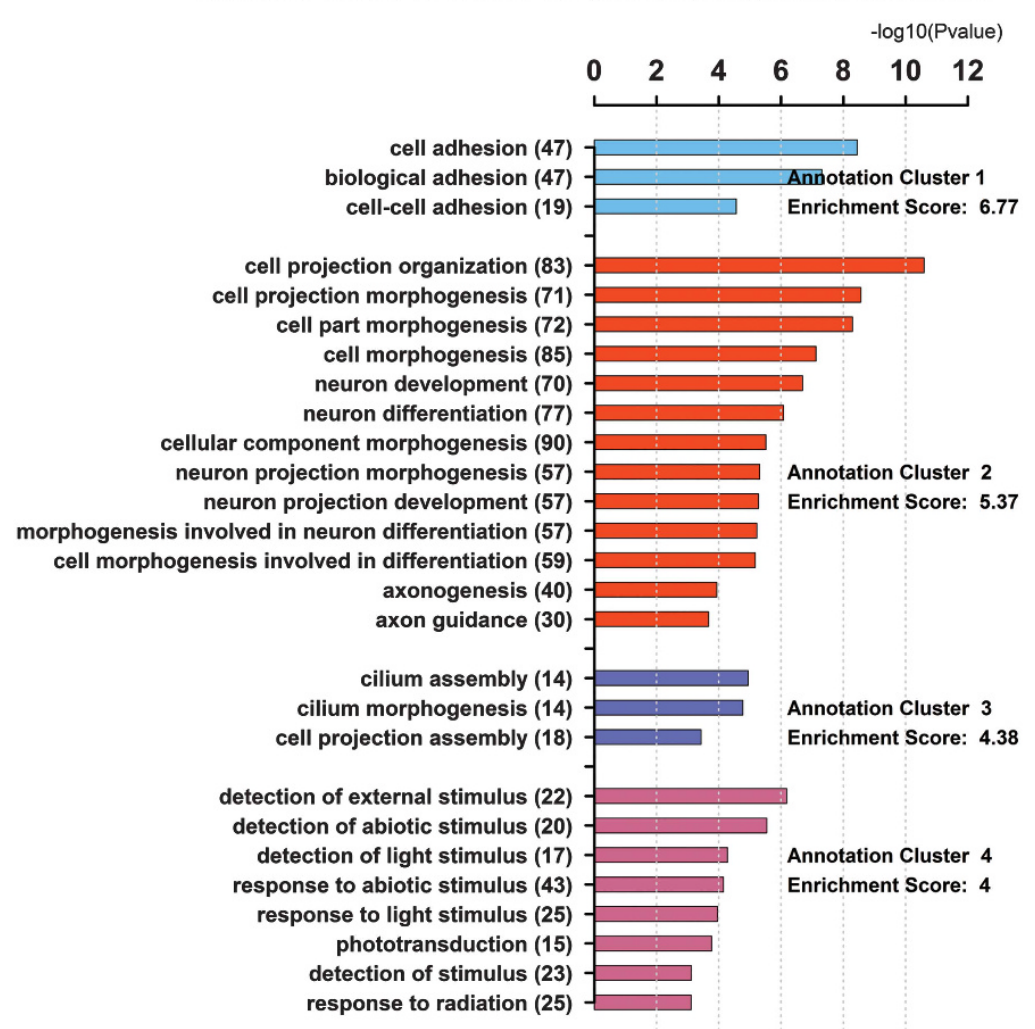

Figure 2 Changes of gene expression during NSCs differentiating to neurons. (a) Scatter plot shows the profiles of gene expression in NSCs and neurons. Significantly DEGs are indicated in colors. Green and red dots are down- and upregulated genes in neurons, respectively. The numbers of DEGs are indicated in the corners. The NSC- and neuronmarker genes (Sca and Elav) are labeled. (b) Read density profile for expression levels of genes Sca and Elav. (c) Significantly enriched GO terms for the DEGs upregulated in neurons. Each set of color bars represent a GO term cluster

Figure S1B). GO term analysis of genes whose NDRs are nucleosome-depleted in NSCs but nucleosomeoccupied in neurons identified enrichment for DNA replication, DNA metabolic process, and so on (Supplementary Figure S3D). In contrast, genes whose NDRs are nucleosome-depleted in neurons but nucleosome-occupied in NSCs are enriched for neuron-related GO terms such as neuron development, dendrite morphogenesis, neuron projection development, and so on (Figure 3e). This suggests that nucleosome remodeling near TSS activates corresponding lineage-commitment genes of each cell type through nucleosome depletion in NDRs during the differentiation. In addition, nucleosome fuzziness around TSS is much higher in NSCs compared with that in neurons (Figure 3f). That is, nucleosome positioning is more delocalized in NSCs than in neurons. 
a

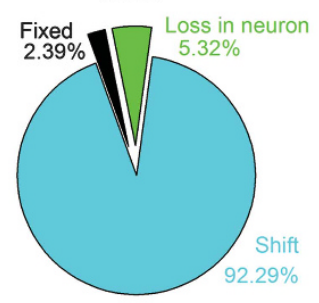

neuron

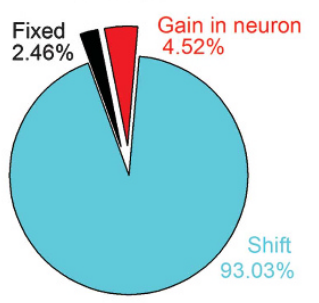

b

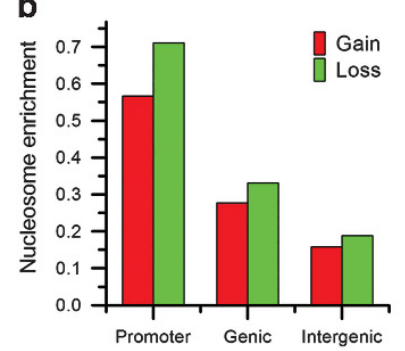

c

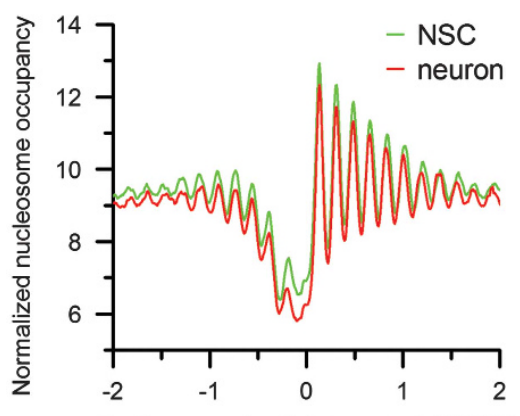

Nucleosome dyad distance to TSS (kb)

e

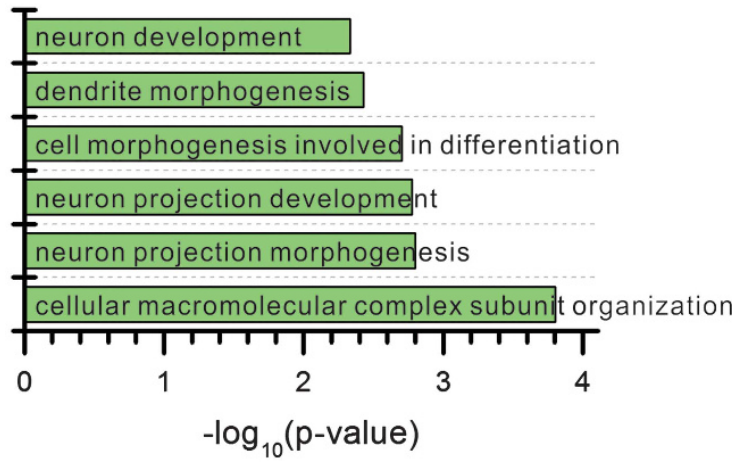

d
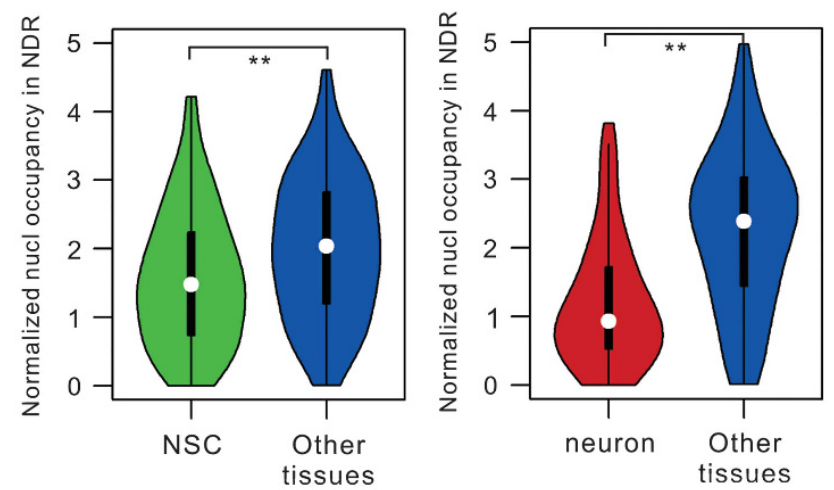

f

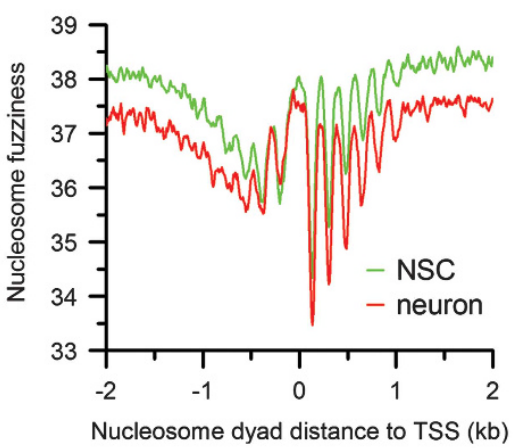

Figure 3 Nucleosome landscape changes. (a) Pie charts show the portions of fixed nucleosome, shift, loss, and gain of nucleosome. Fixed nucleosomes share the same positions in NSCs and neurons. Shift nucleosomes include the nucleosome pairs from NSCs and neurons with overlapping $20 \mathrm{bp}$ or more. Nucleosome loss and gain consists of the rest of nucleosomes. (b) Enrichment of nucleosome gain and loss in different genomic features. The enrichment of nucleosome in promoters equals the number of nucleosomes in promoters normalized by the length of promoters. It is calculated in the same way for genic and intergenic regions. (c) Distribution of nucleosome locations relative to TSSs in the two cell types. Distances to TSS were binned in 10-bp intervals, then nucleosomal read count in each bin was normalized to the total number of uniquely mapped reads in millions, and finally plotted as a smoothed distribution using a moving average of three bins. (d) Nucleosome occupancy in the promoter NDRs (-200 and +50 bp to TSSs) in NSC-, neuron-, and other tissue-specific genes $\left({ }^{* *} P<0.01\right.$, Wilcoxon's rank-sum test). Other tissues include all the non-neuron tissues in Figure 1c and non-NSC tissues in Supplementary Figure S1B, respectively. (e) Significantly enriched GO terms for the genes with a promoter NDR in neurons. (f) Distribution of nucleosome fuzziness relative to TSSs. Fuzziness measures delocalization of a nucleosome positioning, which is calculated as the standard deviation of all read coordinates that contribute to a nucleosome location

Dynamics of HMs in promoter regions. HMs function in many fundamental biological processes. ${ }^{21}$ To understand how HMs alter and the resultant influence on gene expression during the differentiation, we first profiled $\mathrm{HM}$ signals in promoter regions ( $\pm 1 \mathrm{~kb}$ of TSS). H3K4me3 levels in promoter regions take on a bimodal distribution (Supplementary Figure S4A). The smallest level value between the two peaks is selected as the threshold. The promoters with H3K4me3 level higher than the threshold are considered marked by $\mathrm{H} 3 \mathrm{~K} 4 \mathrm{me}$. The thresholds for other HMs were determined in a similar manner (see Materials and Methods for details). Thus, HM status in each promoter is defined by combinatorial HMs. Bivalent promoters (H3K4me3+ and $\mathrm{H} 3 \mathrm{~K} 27 \mathrm{me} 3+)$ are prevalent in mammalian cells. ${ }^{7,22-24}$ However, we only observed a very small number of bivalent promoters in both cell types (Supplementary Figure S4B). This suggests that $\mathrm{H} 3 \mathrm{~K} 4 \mathrm{me} 3$ and H3K27me3 are generally exclusive in promoter regions of Drosophila NSCs and neuronal cells. This is consistent with the previous finding that bivalency is not a common feature of fly embryo epigenome. ${ }^{25}$ Nevertheless, 
a

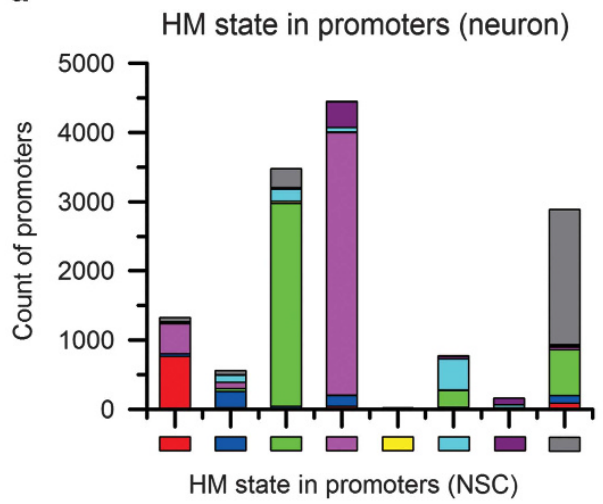

d

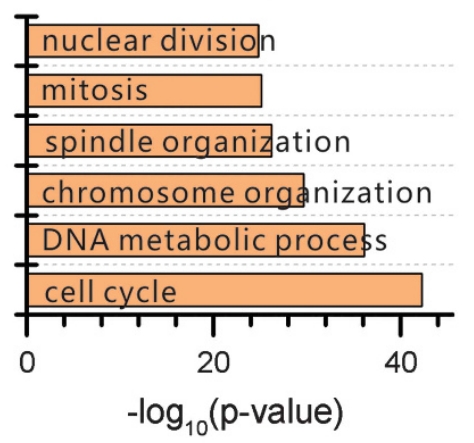

None

$\mathrm{K} 4 \mathrm{me} / \mathrm{K} 4 \mathrm{me} 1 / \mathrm{K} 27 \mathrm{me} 3$

$\mathrm{K} 4 \mathrm{me} 1 / \mathrm{K} 27 \mathrm{me} 3$

$\mathrm{K} 4 \mathrm{me} / \mathrm{K} 4 \mathrm{me} 1$

$\mathrm{K} 27 \mathrm{me} 3$

$\mathrm{K} 4 \mathrm{me} 1$

$\mathrm{K} 4 \mathrm{me} 3$
$\mathrm{K} 4 \mathrm{me} / \mathrm{K} 27 \mathrm{me} 3$

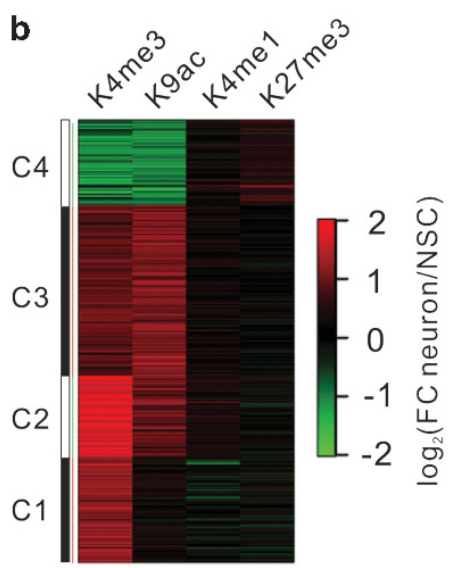

c

C2

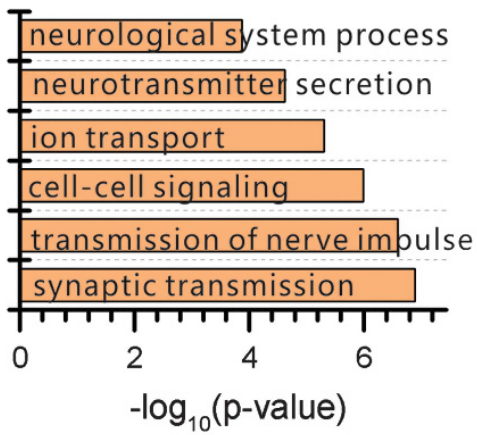

nSC $\square$ neuron

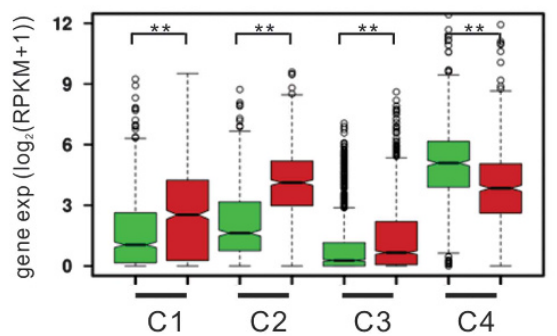

neulopeptide signaling pathway

neurdlogical system process

ion transport

C3

cell surface receptor linked signal transduction

sensory perception of smell

G-protein coupled receptor protein signaling pathway

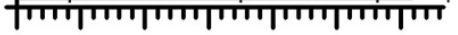

$$
\begin{array}{llccccc}
0 & 2 & 4 & 6 & 8 & 10 & 12 \\
-\log _{10}(p \text {-value }) &
\end{array}
$$

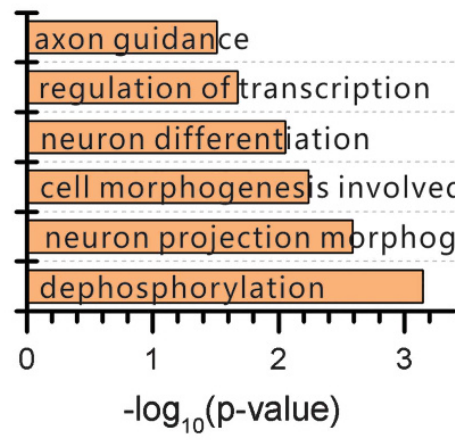

e

H3K4me3

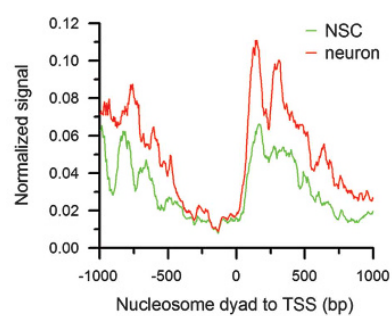

H3K9ac

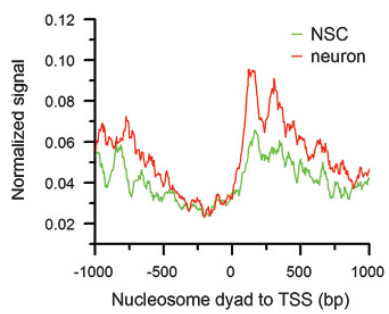

$\mathrm{H} 3 \mathrm{~K} 4 \mathrm{me} 3$

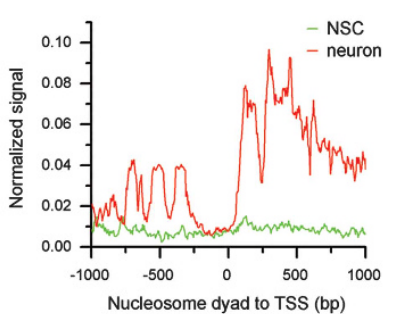

C1

differentiation

Figure 4 HM dynamics in promoter regions. (a) The count of each category of promoters defined by HM state in NSCs changing to other categories in neurons. (b) Clustering view of $\mathrm{HM}$ fold change in the promoters containing at least one $\mathrm{HM}$ with fold change $\geqslant 2$. (c). Genes in the four clusters in (b) are significantly differentially expressed between NSCs and neurons ( ${ }^{* *} P<0.01, T$-test). (d) Significantly enriched GO BP terms for the four categories of promoters in (b). (e) Increase in existing H3K4me3 and H3K9ac signals from NSCs to neurons in the promoters of gene set 'neurological system process' (GO:0050877). (f) Track view of HM dynamics and the concordant expression changes of the sample gene Bchs for (e). (g) De novo gain of H3K4me3 and H3K9ac from NSCs to neurons in the promoters of gene set 'transmission of nerve impulse' (GO:0019226). (h) Track view of HM dynamics and the concordant expression changes of the sample gene Syn for ( $g$ ) 

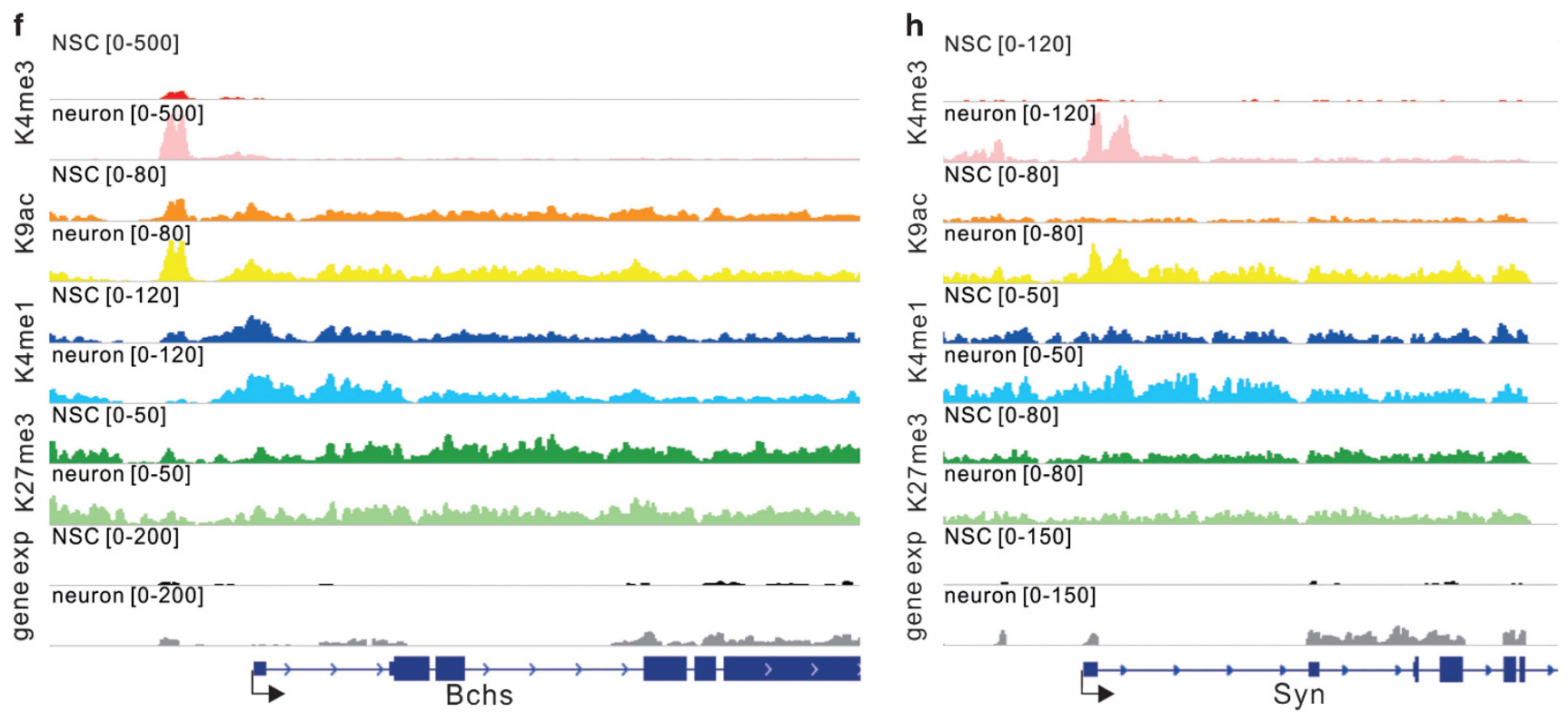

Figure 4 Continued

HM states in promoters serve as a predictive for the gene activity. Genes marked by active H3K4me3 have the highest expression levels, whereas genes marked by repressive H3K27me3 have the lowest expression levels. Expression levels of genes marked by covalent HMs overall are positively correlated to the portion of active mark(s) (Supplementary Figure S4C). A recent study showed that H3K4me1 in promoters has a role in gene repression in diverse mammalian cell types. ${ }^{26}$ Genes with H3K4me1 and H3K27me3 covalent marks have a very low expression level in our study. GO analysis of these genes identified enrichment for gut development, leg disc development, head segmentation, and other tissue-specific commitment (Supplementary Figures S4C and D). This indicates that the covalent $\mathrm{H} 3 \mathrm{~K} 4 \mathrm{me} 1$ and $\mathrm{H} 3 \mathrm{~K} 27 \mathrm{me} 3$ in promoters contribute to repression of non-neural tissue genes.

To understand how HMs change in promoters during the differentiation, we categorized promoters by $\mathrm{HM}$ content in neurons transited from promoters with a certain HM state in NSCs. The results show that the majority of promoters maintain their HM state during differentiation (Figure 4a). We further focused on the genes with one or more of HMs in their promoters that change by at least twofolds. Clustering analysis obtained four patterns of HM changes in promoters (Figure 4b). A set of genes show great decrease in active marks H3K4me3 and H3K9ac (Cluster 4). Consequently, gene expression levels are significantly downregulated in neurons (Figure 4c). These genes are enriched on the pluripotencyrelated GO terms such as cell cycle, DNA metabolic process, and chromatin organization (Figure $4 d$ ). On the contrary, the other two sets of genes exhibit prominent increase in H3K4me3 and H3K9ac (Clusters 3 and 2). The last set of genes show prominent increase in $\mathrm{H} 3 \mathrm{~K} 4 \mathrm{me} 3$ and intermediate decrease in $\mathrm{H} 3 \mathrm{~K} 4 \mathrm{me} 1$ and $\mathrm{H} 3 \mathrm{~K} 27 \mathrm{me} 3$ (Cluster 1) (Figure 4b). Consistently, expression levels of these three gene sets are significantly higher in neurons than in NSCs (Figure 4c). These genes are enriched on $\mathrm{GO}$ terms related to neuron morphogenesis, differentiation, and function (Figure 4d).

The augmented $\mathrm{H} 3 \mathrm{~K} 4 \mathrm{me} 3$ and $\mathrm{H} 3 \mathrm{~K} 9 \mathrm{ac}$ signals during the differentiation could be elevated from existing marks in NSCs or de novo established in neurons. It is unclear if these two modes are associated with different functions. To answer this question, we examined $\mathrm{HM}$ changes in the promoters of different gene sets classified by GO functional annotations. Intriguingly, genes associated with functions related to neuron development gain H3K4me3 and H3K9ac in promoters upon differentiation by increase from their existing levels in NSCs (Figures 4e and f). In contrast, genes associated with neuronal functions gain $\mathrm{H} 3 \mathrm{~K} 4 \mathrm{me} 3$ and $\mathrm{H} 3 \mathrm{~K} 9 \mathrm{ac}$ in promoters upon differentiation through de novo deposition (Figures $4 \mathrm{~g}$ and $\mathrm{h}$ ). The repressive marks (H3K4me1 and H3K27me3) remain at an unchanged low level (Supplementary Figures S4E and F). Conversely, H3K4me3 and $\mathrm{H} 3 \mathrm{~K} 9 \mathrm{ac}$ marks greatly decrease during the differentiation in genes associated with neural pluripotency, for example, notch signaling pathway (Supplementary Figures S4G and $\mathrm{H}$ ). This suggests that different chromatin remodeling modes in promoter regions regulate the activity of genes with distinct function themes.

Chromatin remodeling at distal regulatory elements. In addition to promoters, enhancers also have key roles in determining the transcriptional profile of a cell through epigenetic regulation. We first identified 7728 enhancers by H3K4me1 signals and classified enhancers by H3K27ac signals: active (H3K4me1+ and $\mathrm{H} 3 \mathrm{~K} 27 \mathrm{ac}+)$, poised (H3K4me1+ and H3K27ac-), and off (H3K4me1-). Approximately $65 \%$ of enhancers are active in both cell types (Supplementary Figure S5A). As expected, expression levels of genes associated with active enhancers are 
a

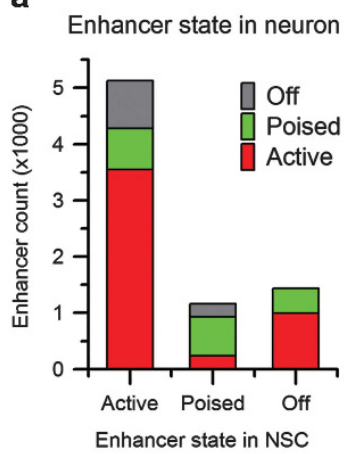

b

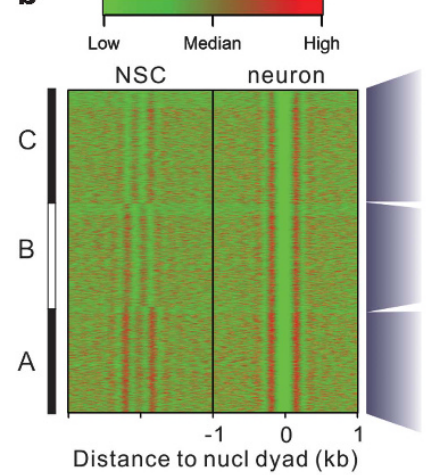

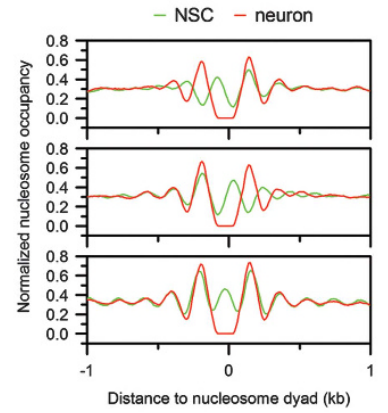

d

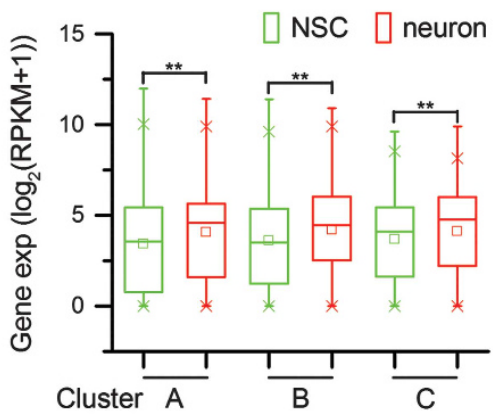

C

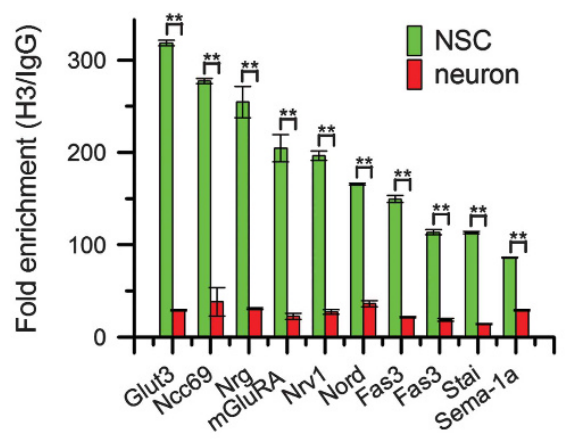

e

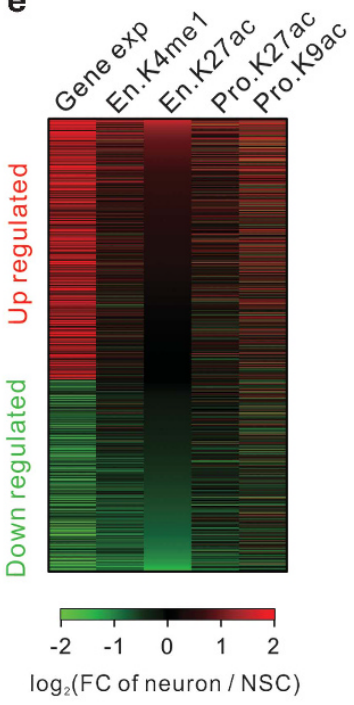

f

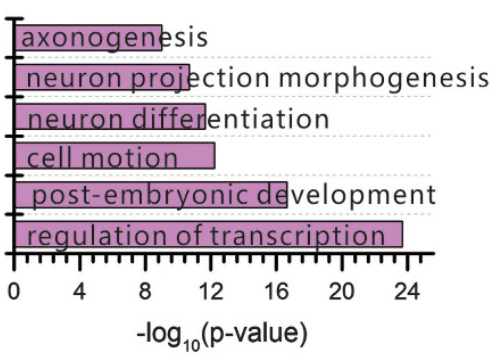

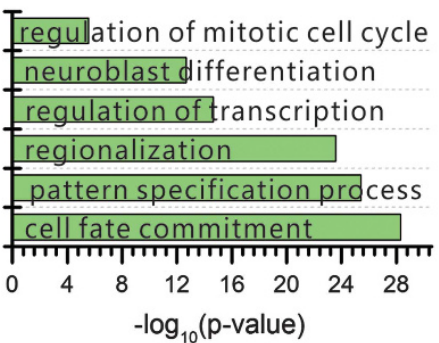

Figure 5 Chromatin remodeling in enhancers. (a) Statistical summary of each category of enhancers defined by HM state in NSCs changing to other categories in neurons. (b) Nucleosome organization in the regions flanking NDRs within neuron enhancers. Left heatmaps show clustering view of nucleosome occupancy in $\pm 1 \mathrm{~kb}$ regions centering at NDRs in neuron enhancers. Right curve plots show the composite distribution of nucleosomes to the midpoint of the evicted nucleosomes in NDRs in neuron enhancers. (c) ChIP-qPCR validation for evicted nucleosomes in the enhancers of selected genes in neurons $\left({ }^{\star \star} P<0.01, T\right.$-test) (d) Expression levels of genes associated with the three clusters of enhancers in (b) ( ${ }^{\star \star} P<0.01, T$-test). (e) Synergistic HM changes in enhancers and promoters. Upregulated genes have increased expression level as En.K27ac signals increase in neurons. Downregulated genes have decreased expression level as En.K27ac signals decrease in neurons. Genes are ordered in a descending order by the $\log 2$-transformed fold change of En.K27ac. Prefixes En indicates enhancer and Pro indicates promoter. (f) Enriched GO terms for the upregulated (pink) and downregulated (green) genes in (e)

significantly higher than in genes associated with poised and off enhancers (Supplementary Figure S5B). We next examined $\mathrm{HM}$ changes in enhancers during the differentiation. The results show that most of the active and poised enhancers in NSCs remain in the same state in neurons (Figure 5a). Seventy percent of NSC off enhancers become active in neurons. GO analysis of neuronal active enhancers transited from NSC poised and off enhancers identified enrichment for neuron differentiation, neuron projection morphogenesis, neuron recognition, and so on (Supplementary Figure S5C).

NDR formation within neuronal enhancers through nucleosome shift and eviction. We also sought to determine whether NDRs form within enhancers during the differentiation, how they form, and their potential functions. We scanned active enhancers in neurons and collected regions that do not contain nucleosomes and are $150 \mathrm{bp}$ or longer. There are total 2342 such regions that are defined as NDRs in enhancers (Figure 5b). In all, 89.5\% of NDRs are 150-250 bp long indicating only one nucleosome size. Intriguingly, clustering analysis of nucleosome occupancy around NDRs by $K$-means $(K=3)$ revealed three patterns of NDR formation: nucleosome eviction and nucleosome shift to $5^{\prime}$ or $3^{\prime}$ direction (Figure 5b). Chromatin immunoprecipitationqPCR (ChIP-qPCR) results confirmed nucleosome eviction in these neuronal enhancers (Figure $5 \mathrm{c}$ and Supplementary Table S1). Consequently, transcription levels of genes associated with these enhancers are significantly increased from NSCs to neurons (Figure 5d). We further examined the state of these enhancers in NSCs and found that $\sim 75 \%$ of them are active in NSCs (Supplementary Figure S5D). This implies that openness by NDR formation in these enhancers in neurons may increase DNA accessibility and have a dominant role in the activation of the target genes. Similarly, 
nucleosome reorganization at functional enhancers exposes conserved regulatory elements in active CD4+ $\mathrm{T}$ cells compared with the resting state. ${ }^{27}$

Chromatin remodeling in promoters and enhancers synergistically activates NSCs differentiating to neurons. To understand how chromatin remodeling in promoters and enhancers work together to regulate the differentiation, we focused on the genes whose expression changes are positively correlated with H3K27ac signal changes during the differentiation. That is, these genes are upregulated when H3K27ac signals increase upon differentiation and vice versa. Remarkably, the results found that chromatin remodeling in both proximal and distal regulatory elements regulate gene expression in a synergetic manner. Namely, active HM contents increase together in both promoters and enhancers to upregulate target genes and vice versa (Figure 5e). GO analysis of the upregulated genes identified enrichment for axonogesis, neuron projection morphogenesis, neuron differentiation, and so on. Conversely, the downregulated genes are enriched for GO terms related to neural pluripotency or progenitor such as regulation of mitotic cell cycle, neuroblast differentiation, and so on (Figure 5f).

\section{Discussion}

Epigenetic alterations have a critical role in cell differentiation. We profiled transcriptome, nucleosome occupancy, and core HMs in affinity-purified NSCs and neuronal cells, respectively. Epigenomics analysis found that nucleosome occupancy decrease in promoter NDRs upregulates the expression of target genes, and NDR formation in promoters facilitates NSCs differentiating into neurons. Remarkably, NDRs form in enhancers during the differentiation in two modes: nucleosome shift and eviction. As a result, it upregulates the expression of target genes and promotes differentiation. Genes gain active H3K4me3 and H3K9ac signals in promoters upon differentiation by de novo deposition in neurons or increase from the existing levels in NSCs. The corresponding gene sets have different neuron-related functions. Conversely, genes that lose active HMs in promoters are related to neural pluripotency and downregulated. In addition, chromatin remodeling in promoters and enhancers takes place and regulates gene expression in a synergistic manner to facilitate NSCs differentiating into neurons. Our study sheds new light on chromatin remodeling patterns and its epigenetic role in in vivo neuron development in early Drosophila embryos.

Activity control of enhancers regulates cell-type-specific patterns of gene expression and has a critical role in development. ${ }^{28-30}$ Nucleosome positioning is one of the key means that control the binding of transcription factors to their motifs. It has been reported that nucleosome-depleted enhancers are important to the activity of cell-type-specific regulators. ${ }^{31}$ Here we found that NDRs form within neuronal enhancers during differentiation. As a result, the target genes associated with these enhancers are upregulated (Figures $5 b$ and d). This implies that NDRs within enhancers may expose cis-regulatory elements and facilitates binding of transcription factors. Such dynamic nucleosome positioning is composed of observations that most enhancers are cell-type-specific. ${ }^{32,33}$
Notably, enhancer establishment (measured by H3K4me1 signal) is initiated earlier and can indicate the differentiation potential of progenitor cells earlier than gene expression profiles during blood formation. ${ }^{6}$ It will be interesting to know whether openness within enhancers (measured by NDR) can serve as an indicator of lineage commitment during in vivo neural development in Drosophila. This goal will be achieved when clean marker genes for distinct intermediate cell types during NSCs differentiating to a wide variety of mature neural cells are available.

HMs have a critical role in the development of embryonic nervous system in Drosophila. NSCs differentiating to glial cells require low levels of H3K9ac. High levels of CREGbinding protein (a histone acetyl-transferase) result in high levels of H3K9ac and disrupt gliogensis in Drosophila embryonic neural development. ${ }^{34}$ In contrast, our results show that H3K9ac signals in promoters are increased in neuronal cells compared with NSCs. This is consistent with the observation that neurons have relatively high levels of H3K9ac compared with most glial cells. ${ }^{34}$ This implies that HM contents in promoters regulate the expression of genes critical for neural cell lineage specification. Intriguingly, we further revealed that the high levels of active H3K4me3 and H3K9ac come from both de novo deposition in neurons and increase from existing levels in NSCs (Supplementary Figures S4E and $\mathrm{G})$. Moreover, these two subclasses of genes are enriched for different neuron-related functions. The findings improve our understanding of epigenetic regulatory mechanisms underlying Drosophila embryonic neuronal differentiation in vivo.

Both promoters and enhancers are critical elements that orchestrate the regulation of gene expression during many biological processes. It has been reported that distal enhancers could be localized in close physical proximity to promoters through the formation of chromatin loops to regulate gene expression during lineage commitment and somatic cellular reprogramming. ${ }^{35,36}$ Our results show that the core HMs in promoters and enhancers concordantly alter to regulate the expression of genes critical for NSCs differentiating to neurons (Figures $5 \mathrm{~d}$ and e). However, it is unclear whether enhancer-promoter looping forms because of the involvement of other transcription factors during differentiation. Future study of genomic architecture dynamics during differentiation through circular chromatin conformation capture (4C)-based methods will fill this gap.

\section{Materials and Methods}

Drosophila strains. The transgenic line $w^{1118} ;$; [UASRG]6 $(I I I)^{15}$ was kindly provided by Professor Steven Henikoff (Fred Hutchinson Cancer Research Center, Seattle, WA, USA). This stock expresses the transgene (NTF) 3xFLAG-BLRP$m$ Cherry-RanGap and BirA under GAL4 driver. Other lines such as Elav-Gal4;Tm3/ Tm6B (X;III), Sca-Gal4 (II) and sp/cyo;Dr./Tm6B (II;III) were obtained from the Bloomington Stock Center (Bloomington, IN, USA).

Elav-Gal4; Tm3/Tm6B virgin flies were crossed with $w^{1118} ;$ p[UASRG]6 male flies to generate the F1 offspring Elav-Gal4/+;p[UASRG]6/Tm3. The F1 male flies were backcrossed with Elav-Gal4;Tm3/Tm6B virgin flies to generate the F2 offsprings Elav-Gal4;p[UASRG]6/Tm3 (female flies) and Elav-Gal4/+;p[UASRG]6/Tm3 (male flies). Then, the F2 offsprings were self-crossed to generate the F3 offsprings that are homozygous, Elav-Gal4;p[UASRG]6 (female flies) and Elav-Gal4/+;p [UASRG]6 (male flies). The embryos of F3 flies were collected by self-crossing to isolate neurons. 
First, Sca-Gal4 virgin flies and sp/cyo;Dr/Tm6B male flies were crossed to generate the $F 1$ offspring. We retained the ones with genotype Sca-Gal4/cyo;+/Tm6B (denoted as F1A) for the next cross. The sp/cyo;Dr/Tm6B virgin flies were crossed with $w^{1118} ; p[$ [USRG]6 male flies to generate the $\mathrm{F} 1$ offspring. We retained the ones with genotype +/cyo;p[UASRG]6/Tm6B (denoted as F1B) for the next cross. Next, $\mathrm{F} 1 \mathrm{~A}$ virgin flies were crossed with sp/cyo; $\mathrm{Dr} / \mathrm{Tm} 6 \mathrm{~B}$ male flies to produce the F2 offspring. We retained the ones with genotype Sca-Gal4/cyo;Dr/Tm6B (denoted as F2A) for the next cross. The sp/cyo; Dr/Tm6B virgin flies were crossed with F1B male flies to generate the F2 offspring. We retained the ones with genotype sp/cyo; $p$ [UASRG]6/Tm6B (denoted as F2B) for the next cross. Later, the F2A virgin flies were crossed with F2B male flies to generate the F3 offspring. We retained the ones with genotype Sca-Gal4/cyo;p[UASRG]6/Tm6B (denoted as F3A) for the next cross. Finally, F3A flies were self-crossed to generate the F4 offspring. We retained the ones with genotype Sca-Gal4/cyo;P[UASRG]6 (denoted as F4A). The embryos of F4A flies were collected by self-crossing to isolate NSCs.

Antibodies. The following antibodies were used for ChIP: H3K4me3 (ab8580; Abcam, Cambridge, MA, USA), H3K4me1 (ab8895; Abcam), H3K9ac (ab10812; Abcam), H3K27ac (ab4729; Abcam) and H3K27me3 (ab6002; Abcam). Antibodies used for immunostaining were as follows: rat- $\alpha$-Elav (from Developmental Studies Hybridoma Bank, lowa City, IA, USA) and goat anti-rabbit IgG H\&L (Alexa Fluor 488; Jackson ImmunoResearch, West Grove, PA, USA). Anti-Flag-coated M2 magnetic beads (Sigma-Aldrich, St. Louis, MO, USA) were used for pull down.

Embryo collection and formaldehyde crosslinking. Embryos were collected on grape juice plates with a yeast paste from embryo collection cages for $2 \mathrm{~h}$, and then aged at $25^{\circ} \mathrm{C}$ for 3 and 10 additional hours. The harvested embryos are 5-7 and 12-14 $\mathrm{h}$ old, respectively. Embryos were transferred onto the mesh with PBST (PBS (137 mM NaCl, $4.3 \mathrm{mM} \mathrm{Na}_{2} \mathrm{HPO}_{4}, 1.4 \mathrm{mM} \mathrm{NaH}_{2} \mathrm{PO}_{4}$ )+0.1\% Triton $X-100)$, and were washed with tap water to remove the yeast. Then, embryos were dechorionated with $50 \%$ hypochloric acid for 3 min and were crosslinked in a 1:3 mixture of ChIP-Fixed buffer $(50 \mathrm{mM}(\mathrm{pH} 7.6)$ HEPES, $100 \mathrm{mM} \mathrm{NaCl}, 0.1 \mathrm{mM}$ EDTA, $0.5 \mathrm{mM}$ EGTA) with $1.8 \%$ formaldehyde and heptane for $15 \mathrm{~min}$ on a shaker with a speed of 300 r.p.m. The aqueous and organic phase was replaced with PBST containing $0.25 \mathrm{mM}$ glycine to cease the crosslinking reaction. The fixed embryos were rinsed three times with PBST and then stored at $-80^{\circ} \mathrm{C}$ for future use.

Purification of tagged nuclei from Drosophila embryos. Purification of tagged nuclei was performed using the INTACT technology as described previously. ${ }^{15,37}$ In brief, $0.3-0.5 \mathrm{~g}$ of fixed embryos were suspended in $4 \mathrm{ml}$ of cold HB125 buffer ( $15 \mathrm{mM} \mathrm{NaCl}, 40 \mathrm{mM} \mathrm{KCl}, 15 \mathrm{mM}$ (pH 7.5) Tris-HCl, $0.125 \mathrm{M}$ sucrose, $0.5 \mathrm{mM}$ spermidine, $0.15 \mathrm{mM}$ spermine, EDTA, $0.5 \mathrm{mM}$ EGTA, $1 \times$ Complete protease inhibitor $(\mathrm{PI}))$ and dounce homogenized. To monitor bead binding $0.5 \mathrm{ml}$ of $5 \mathrm{mg} / \mathrm{ml}$ DAPI solution was added. The nuclei mixture was filtered through one layer of Miracloth into $50 \mathrm{ml}$ conical tube and diluted to $40 \mathrm{ml}$ with cold HB125 buffer; afterwards, $3 \mathrm{ml}$ of OptiPrep (Sigma-Aldrich) was added and then centrifuged at $1000 \times g$ for $10 \mathrm{~min}$ at $4^{\circ} \mathrm{C}$. The supernatant and OptiPrep cushion were discarded, leaving $\sim 2 \mathrm{ml}$ of $\mathrm{HB} 125$ containing nuclei concentrated at the interface. Isolated nuclei were suspended in HB125 with $60 \mu \mathrm{l}$ of anti-Flag M2 magnetic beads slurry and incubated on a rotator for $2 \mathrm{~h}$ at $4{ }^{\circ} \mathrm{C}$. Beads with affinity-bound nuclei were absorbed by the magnet and then washed three times using HB125. Purified nuclei were stored at $-80^{\circ} \mathrm{C}$ before proceeding with ChIP.

Immunofluorescence microscopy. Embryos were dechorionated in 50\% solution of bleach and fixed in a $1: 1$ mixture of $4 \%$ formaldehyde in PBS with $0.3 \%$ Tween-20 and heptane for $20 \mathrm{~min}$ on a shaker. The aqueous phase was discarded and replaced with methanol, and embryos were shaken for 3-5 min at 300 r.p.m. to burst vitelline membranes. Embryos were rinsed three times with methanol and then rinsed three times with PBS containing $0.3 \%$ Triton X-100. Fixed embryos were blocked with PBST supplemented by $5 \%$ normal donkey serum (NDS) for 30 min and then incubated with primary antibodies of various dilutions in PBST containing $5 \%$ NDS overnight at $4{ }^{\circ} \mathrm{C}$. These embryos were washed as described above, followed by a $1-2 \mathrm{~h}$ secondary antibody incubation, and then were washed three times again to avoid nonspecific binding. These stained embryos were mounted on slides with additional $50 \%$ glycerol in PBS. Slides were examined on a Zeiss Imager M2 microscopy (Goettingen, Germany). To examine bead binding, DAPI-stained total and affinity-purified nuclei were counted on a hemacytometer, respectively.
MNase-seq and ChIP-seq. Affinity-purified nuclei were pelleted by centrifugation at $1000 \times g$ for 5 min and suspended with $500 \mu$ l of $37^{\circ} \mathrm{C}$ preheated MNase (micrococcal nuclease) digestion buffer (10 mM (pH 7.5) Tris- $\mathrm{HCl}, 15 \mathrm{mM}$ $\mathrm{NaCl}, 60 \mathrm{mM} \mathrm{KCl}, 2 \mathrm{mM} \mathrm{CaCl}, 0.15 \mathrm{mM}$ spermine, $0.5 \mathrm{mM}$ spermidine, $1 \times \mathrm{Pl}$ ) with $12 \mathrm{U}$ MNase (Worthington Biochemical Corporation, Lakewood, NJ, USA) and incubated at $37^{\circ} \mathrm{C}$ for $20 \mathrm{~min}$. The reaction was terminated on ice by adding EDTA to a final concentration of $10 \mathrm{mM}$ for $10 \mathrm{~min}$. The supernatant was discarded and the pellet was washed with A2 buffer ( $140 \mathrm{mM} \mathrm{NaCl}, 15 \mathrm{mM}$ (pH 7.6) HEPES, $1 \mathrm{mM}$ EDTA, $0.5 \mathrm{mM}$ EGTA, $0.1 \%$ Triton $\mathrm{X}-100,0.1 \%$ sodium deoxycholate, $1 \times \mathrm{Pl}$ ) and resuspended in A2 buffer with $0.1 \%$ SDS. The nucleosome pellet was dissolved through sonication with three cycles of $20 \mathrm{~s}$ duration with at least $40 \mathrm{~s}$ pauses between cycles at the power setting of 6 (out of 20) on a Misonix sonicator XL-2000 (Newtown, CT, USA). The supernatants with chromatin were kept for the next ChIP assay, or were reverse crosslinked to harvest nucleosomal DNA fragments as follows: chromatin was treated with RNase $\mathrm{A}$ at $37^{\circ} \mathrm{C}$ for $0.5-1 \mathrm{~h}$ and followed by proteinase $\mathrm{K}$ treatment at $65^{\circ} \mathrm{C}$ for $2 \mathrm{~h}$. Later, the nucleosomal DNA was retracted by phenol-chloroform and precipitated with a 1:10:100:200 mixture of $20 \mathrm{mg} / \mathrm{ml}$ glycogen, $3 \mathrm{M}$ (pH 5.3) NaOAc, nucleosomal DNA mixture, and cold 100\% ethanol.

Chromatin of 10-15 $\mu \mathrm{g}$ was used for each ChIP reaction with HM antibodies of various doses as described in the specifications. Mixture containing chromatin, antibody, and ChIP buffer (16.7 mM (pH 8.1) Tris-HCl, $167 \mathrm{mM} \mathrm{NaCl}, 1.2 \mathrm{mM}$ EDTA, $1 \%$ Triton X-100, $0.01 \%$ SDS) was incubated overnight on a rotator at $4{ }^{\circ} \mathrm{C}$. Then, $20 \mu \mathrm{l}$ of ChIP-Grade Protein G Magnetic Beads (Cell Signaling; no. 9006) was added to each IP reaction. The mixture was incubated for $2 \mathrm{~h}$ with rotation. Afterwards, beads were washed three times with low salt wash buffer (2 mM EDTA, $20 \mathrm{mM}$ (pH 8.1) Tris-HCl, $0.1 \%$ SDS, $1 \%$ Triton X-100, $150 \mathrm{mM} \mathrm{NaCl}$ ) and once with high salt wash buffer (2 mM EDTA, $20 \mathrm{mM}(\mathrm{pH}$ 8.1) Tris-HCl, $0.1 \%$ SDS, $1 \%$ Triton X-100, $500 \mathrm{mM} \mathrm{NaCl}$ ) for $5 \mathrm{~min}$ each wash. Beads were suspended in $150 \mu \mathrm{l}$ of ChIP elution buffer (50 mM (pH 8.1) Tris-HCl, $10 \mathrm{mM} \mathrm{EDTA}, 0.1 \% \mathrm{SDS}$ ) at $65^{\circ} \mathrm{C}$ for $45 \mathrm{~min}$.

The purified mononucleosomal DNA was subjected to massively parallel DNA sequencing on Illumina HiSeq2000 platform (San Diego, CA, USA) using a 49 bp single-end protocol.

Nuclear RNA-seq analysis. Nuclear RNA was isolated form tagged nuclei of the affinity-purified embryos without crosslinking using the RNeasy Micro Kit (Qiagen, Valencia, CA, USA). Genomic DNAs were removed with Turbo DNA-Free Kit (Ambion, Waltham, MA, USA). The RNA sequencing libraries were constructed using standard Illumina libraries prep protocols. RNA-seq was performed on Illumina HiSeq2000 platform. Sequencing reads were aligned to the Drosophila transcripts (FlyBase r5.43) using TopHat (v.1.3.1) with default parameter setting. ${ }^{38}$ The uniquely mapped reads were assembled into transcripts guided by reference annotation with Cuffdiff (v.1.3.0) ${ }^{38}$ to calculate gene expression levels that were normalized as fragment per kilobase per million mapped fragments. The DEGs were identified with FDR $<0.05$.

Tissue-specific gene lists. Tissue-specific gene lists were downloaded from the Berkeley Drosophila Genome Project in situ database BDGP. ${ }^{19}$ Genes expressed at stages 11-12 and 13-16 in different tissue-specific cells were collected.

Prediction of nucleosome positioning and analysis of dynamic positioning. Nucleosomal sequencing reads were aligned to the Drosophila reference genome $(\mathrm{dm} 3)$ using Bowtie, ${ }^{39}$ allowing maximal two mismatches. The uniquely mapped reads were used to identify genome-wide nucleosome positions through the peak-calling tool GeneTrack ${ }^{20}$ that also calculated read count for each nucleosome. The read count was normalized by total uniquely mapped reads as the nucleosome occupancy. Nucleosome fuzziness was calculated as the standard deviation of the coordinates of all reads defining the same nucleosome as described previously. ${ }^{40}$ It measures how delocalized a nucleosome position is. Each nucleosome was assigned to either of promoter, genic, or intergenic regions depending on in which region the midpoint of the nucleosome was located.

Nucleosome organization change around the TSS was analyzed as in the previous study. ${ }^{40}$ Briefly, Drosophila transcript annotation was downloaded from FlyBase release 5.43. Nucleosomes located within $\pm 1 \mathrm{~kb}$ of TSSs were collected. Nucleosome length equals to the fuzziness value and centers at the nucleosome midpoint that defines the nucleosome position. The region that a nucleosome length spreads out has the nucleosome occupancy. The difference in nucleosome organization around TSSs was measured by subtracting nucleosome occupancy in 
NSCs from that in neurons for each site. The difference clustered by $K$-means $(K=5)$ and plotted as heatmap.

The original composite distribution of nucleosome around TSS of the five clusters of genes in NSCs and neurons, respectively, was calculated by aggregating nucleosomal read count at each distance relative to the TSS as follows: each read represents a nucleosome by extending toward $3^{\prime}$ end to a length of $147 \mathrm{bp}$. The midpoint of extended read defines the nucleosome position. We summed total read counts at each site within $\pm 1 \mathrm{~kb}$ of TSSs for the five gene clusters, respectively. The nucleosome occupancy equals to the read count normalized as RPKM. We further binned the nucleosome occupancy by a 5-bp interval of nucleosome distance to TSS, and smoothed it with 5-bin moving average and 1-bin step size.

Analysis of HM changes in the promoters. Sequencing reads were mapped to the reference genome similar to the above nucleosomal read mapping. We defined promoters as the region $\pm 1 \mathrm{~kb}$ of TSSs. HM levels in a promoter were calculated as all reads within the promoter and normalized as RPKM. The density distribution of H3K4me1, H3K4me3 and H3K9ac levels in promoters is a bimodal pattern. The levels at the valley were chosen as thresholds, which was 11.6 for H3K4me1, 16 for H3K4me3, and 16 for H3K9ac. For qualitative analysis, promoters with a higher level of a certain $\mathrm{HM}$ compared with the threshold are characterized as marked by this HM, otherwise not. The density distribution of H3K27me3 levels in promoters is a normal distribution. We grouped promoters into two classes with or without H3K27me3 by $K$-means clustering $(K=2)$.

The composite distribution of HM around TSS was calculated in the same manner as nucleosome distribution described above.

Chromatin state in enhancers. Enhancers and their chromatin states were determined as described previously. ${ }^{6}$ In brief, H3K4me1-enriched regions (peaks) were identified using $\mathrm{HOMER}^{41}$ using a 1000-bp sliding window with a false discovery rate of $0.1 \%$. In addition, two adjacent peaks need to be separated by at least $1000 \mathrm{bp}$ to avoid redundant detection. We next combined such valid peaks from two cell types as one set of peaks by merging overlapped peaks. The peak with highest HOMER score replaces the overlapped peaks. Then, we retained total 14234 H3K4me1 peaks for the two cell types. To remove false enhancers, we calculated H3K4me3 signals in H3K4me1 peaks and observed a bimodal distribution. A Two Gaussian mixture model was used to fit the distribution to select a threshold $(=4.55)$. The H3K4me1 peaks with H3K4me3 levels higher than the threshold were discarded. It resulted in $7728 \mathrm{H} 3 \mathrm{~K} 4 \mathrm{me} 1$ peaks as the final set of enhancers.

To define chromatin states for enhancers, we first recalculated H3K4me1 and H3K27ac signals in the enhancers for the two cell types. We also randomly selected the same number (7728) of 1000-bp genomic regions and calculated H3K4me1 and H3K27ac signals. The smallest level of the top 10\% signals was used as the falsepositive threshold, which was 5.5 for $\mathrm{H} 3 \mathrm{~K} 4 \mathrm{me} 1$ and 4.5 for H3K27ac, respectively. Enhancers are 'on' with H3K4me1 level higher compared with 5.5 and 'off' otherwise. Of 'on' enhancers, those are 'active' with H3K27ac level higher compared with 4.5 and 'poised' otherwise. Finally, each enhancer was associated with a single gene based on the nearest RefSeq TSS.

Data accession numbers. The RNA-seq, MNase-seq, and ChIP-seq data sets have been deposited in the Gene Expression Omnibus under the accession number GSE80458.

\section{Conflict of Interest}

The authors declare no conflict of interest.

Acknowledgements. We are grateful to Professor Steve Henicoff (Fred Hutchinson Cancer Research Center) for kindly providing Drosophila stock $w^{1118} ; p$ [UASRG]6 (III). This work was supported by the Ministry of Science and Technology of China Grant number 2016YFA0100400 (to CJ) and National Natural Science Foundation of China Grant Nos 91519309 and 31271373 (to CJ).

1. Lister R, Mukamel EA, Nery JR, Urich M, Puddifoot CA, Johnson ND et al. Global epigenomic reconfiguration during mammalian brain development. Science 2013; 341 : 1237905

2. Gaspar-Maia A, Alajem A, Meshorer E, Ramalho-Santos M. Open chromatin in pluripotency and reprogramming. Nat Rev Mol Cell Biol 2011; 12: 36-47.
3. Keohane AM, O'Neill L P, Belyaev ND, Lavender JS, Turner BM. X-Inactivation and histone H4 acetylation in embryonic stem cells. Dev Biol 1996; 180: 618-630.

4. Lee JH, Hart SR, Skalnik DG. Histone deacetylase activity is required for embryonic stem cell differentiation. Genesis 2004; 38: 32-38.

5. Martens JH, O'Sullivan RJ, Braunschweig U, Opravil S, Radolf M, Steinlein P et al. The profile of repeat-associated histone lysine methylation states in the mouse epigenome. EMBO J 2005; 24: 800-812.

6. Lara-Astiaso D, Weiner A, Lorenzo-Vivas E, Zaretsky I, Jaitin DA, David E et al. Chromatin state dynamics during blood formation. Science 2014; 345: 943-949.

7. Mikkelsen TS, Ku M, Jaffe DB, Issac B, Lieberman E, Giannoukos G et al. Genome-wide maps of chromatin state in pluripotent and lineage-committed cells. Nature 2007; 448: 553-560.

8. Li Z, Gadue P, Chen K, Jiao Y, Tuteja G, Schug $\mathrm{J}$ et al. Foxa2 and H2A.Z mediate nucleosome depletion during embryonic stem cell differentiation. Cell 2012; 151 1608-1616.

9. Zheng S, Sun M, Zhang K, Gu J, Guo Z, Tian S et al. Profiling post-translational modifications of histones in neural differentiation of embryonic stem cells using liquid chromatography-mass spectrometry. J Chromatogr B 2016; 1017-1018: 36-44.

10. Sugino K, Hempel CM, Miller MN, Hattox AM, Shapiro P, Wu C et al. Molecular taxonomy of major neuronal classes in the adult mouse forebrain. Nat Neurosci 2006; 9: 99-107.

11. Emmert-Buck MR, Bonner RF, Smith PD, Chuaqui RF, Zhuang Z, Goldstein SR et al. Laser capture microdissection. Science 1996; 274: 998-1001.

12. Deal RB, Henikoff S. A simple method for gene expression and chromatin profiling of individual cell types within a tissue. Dev Cell 2010; 18: 1030-1040.

13. Amin NM, Greco TM, Kuchenbrod LM, Rigney MM, Chung MI, Wallingford JB et al. Proteomic profiling of cardiac tissue by isolation of nuclei tagged in specific cell types (INTACT). Development 2014; 141: 962-973.

14. Henry GL, Davis FP, Picard S, Eddy SR. Cell type-specific genomics of Drosophila neurons. Nucleic Acids Res 2012; 40: 9691-9704.

15. Steiner FA, Talbert PB, Kasinathan S, Deal RB, Henikoff S. Cell-type-specific nuclei purification from whole animals for genome-wide expression and chromatin profiling. Genome Res 2012; 22: 766-777.

16. Robinow S, White K. Characterization and spatial distribution of the ELAV protein during Drosophila melanogaster development. J Neurobiol 1991; 22: 443-461.

17. Campos AR, Rosen DR, Robinow SN, White K. Molecular analysis of the locus elav in Drosophila melanogaster. a gene whose embryonic expression is neural specific. EMBO J 1987; 6: 425-431.

18. Mlodzik M, Baker NE, Rubin GM. Isolation and expression of scabrous, a gene regulating neurogenesis in Drosophila. Genes Dev 1990; 4: 1848-1861.

19. Tomancak P, Berman BP, Beaton A, Weiszmann R, Kwan E, Hartenstein V et al. Global analysis of patterns of gene expression during Drosophila embryogenesis. Genome Bio 2007; 8: R145

20. Albert I, Wachi S, Jiang C, Pugh BF. GeneTrack - a genomic data processing and visualization framework. Bioinformatics 2008; 24: 1305-1306.

21. Kouzarides T. Chromatin modifications and their function. Cell 2007; 128: 693-705.

22. Bernstein BE, Mikkelsen TS, Xie X, Kamal M, Huebert DJ, Cuff $J$ et al. A bivalent chromatin structure marks key developmental genes in embryonic stem cells. Cell 2006; 125: 315-326.

23. Pan G, Tian S, Nie J, Yang C, Ruotti V, Wei H et al. Whole-genome analysis of histone H3 lysine 4 and lysine 27 methylation in human embryonic stem cells. Cell Stem Cell 2007; 1 299-312.

24. Zhao XD, Han X, Chew JL, Liu J, Chiu KP, Choo A et al. Whole-genome mapping of histone $\mathrm{H} 3 \mathrm{Lys} 4$ and 27 trimethylations reveals distinct genomic compartments in human embryonic stem cells. Cell Stem Cell 2007; 1: 286-298.

25. Schuettengruber B, Ganapathi M, Leblanc B, Portoso M, Jaschek R, Tolhuis B et al. Functional anatomy of polycomb and trithorax chromatin landscapes in Drosophila embryos. PLOS Biol 2009; 7: e13.

26. Cheng J, Blum R, Bowman C, Hu D, Shilatifard A, Shen S et al. A role for H3K4 monomethylation in gene repression and partitioning of chromatin readers. Mol Cell 2014; 53: 979-992.

27. Schones DE, Cui K, Cuddapah S, Roh TY, Barski A, Wang Z et al. Dynamic regulation of nucleosome positioning in the human genome. Cell 2008; 132: 887-898.

28. Bulger M, Groudine M. Functional and mechanistic diversity of distal transcription enhancers Cell 2011; 144: 327-339.

29. Hawrylycz MJ, Lein ES, Guillozet-Bongaarts AL, Shen EH, $\mathrm{Ng} \mathrm{L}$, Miller JA et al. An anatomically comprehensive atlas of the adult human brain transcriptome. Nature 2012; 489: 391-399.

30. Maston GA, Evans SK, Green MR. Transcriptional regulatory elements in the human genome. Annu Rev Genom Hum Genet 2006; 7: 29-59.

31. Taberlay PC, Statham AL, Kelly TK, Clark SJ, Jones PA. Reconfiguration of nucleosomedepleted regions at distal regulatory elements accompanies DNA methylation of enhancers and insulators in cancer. Genome Res 2014; 24: 1421-1432.

32. Heintzman ND, Hon GC, Hawkins RD, Kheradpour $P$, Stark A, Harp LF et al. Histone modifications at human enhancers reflect global cell-type-specific gene expression. Nature 2009; 459: 108-112.

33. Heintzman ND, Ren B. The gateway to transcription: identifying, characterizing and understanding promoters in the eukaryotic genome. Cell Mol Life Sci 2007; 64: 386-400. 
34. Flici H, Erkosar B, Komonyi O, Karatas OF, Laneve P, Giangrande A. Gcm/Glide-dependent conversion into glia depends on neural stem cell age, but not on division, triggering a chromatin signature that is conserved in vertebrate glia. Development 2011; 138: 4167-4178.

35. Apostolou E, Hochedlinger K. Chromatin dynamics during cellular reprogramming. Nature 2013; 502: 462-471.

36. Shchuka VM, Malek-Gilani N, Singh G, Langroudi L, Dhaliwal NK, Moorthy SD et al. Chromatin dynamics in lineage commitment and cellular reprogramming. Genes 2015; 6: 641-661.

37. Deal RB, Henikoff S. The INTACT method for cell type-specific gene expression and chromatin profiling in Arabidopsis thaliana. Nat Protoc 2011; 6: 56-68.

38. Trapnell C, Roberts A, Goff L, Pertea G, Kim D, Kelley DR et al. Differential gene and transcript expression analysis of RNA-seq experiments with TopHat and Cufflinks. Nat Protoc 2012; 7: 562-578.

39. Langmead B, Trapnell C, Pop M, Salzberg SL. Ultrafast and memory-efficient alignment of short DNA sequences to the human genome. Genome Biol 2009; 10: R25.

40. Shi J, Zheng M, Ye Y, Li M, Chen X, Hu X et al. Drosophila Brahma complex remodels nucleosome organizations in multiple aspects. Nucleic Acids Res 2014; 42: 9730-9739.
41. Heinz S, Benner C, Spann N, Bertolino E, Lin YC, Laslo P et al. Simple combinations of lineage-determining transcription factors prime cis-regulatory elements required for macrophage and B cell identities. Mol Cell 2010; 38: 576-589.

(c) (i) This work is licensed under a Creative Commons Attribution 4.0 International License. The images or other third party material in this article are included in the article's Creative Commons license, unless indicated otherwise in the credit line; if the material is not included under the Creative Commons license, users will need to obtain permission from the license holder to reproduce the material. To view a copy of this license, visit http:/l creativecommons.org/licenses/by/4.0/

(C) The Author(s) 2017

Supplementary Information accompanies this paper on Cell Death and Differentiation website (http://www.nature.com/cdd) 